\title{
Experiência no ensino de patologia em tempos de pandemia
}

\author{
Experience in teaching pathology during the pandemic
}

\begin{tabular}{r|r} 
Fabiana Toledo Bueno Pereira' (1) & fabiana.pereira@fcmsantacasasp.edu.br \\
Fabiola Del Carlo Bernardi' (D) & fabiola.bernardi@fcmsantacasasp.edu.br \\
Geanete Pozzan' (1) & geanete.pozzan@fcmsantacasasp.edu.br
\end{tabular}

\section{RESUMO}

Introdução: A patologia é uma disciplina básica que exerce o link entre ciclos básicos e clínico-cirúrgicos. A partir do início de 2020, com a pandemia provocada pela síndrome respiratória aguda grave do coronavírus 2 (severe acute respiratory syndrome coronavirus 2 - Sars-CoV-2) e a implantação do ensino remoto emergencial (ERE), o curso de patologia em nossa instituição sofreu alterações e adaptações. O presente trabalho tem como objetivos relatar as atividades desenvolvidas em nossa faculdade para o ensino da patologia geral, dentro do contexto do ERE, e discutir como parte dessas estratégias poderá ser incorporada após o término da pandemia.

Relato de experiência: Para o ensino das alterações macroscópicas, utilizamos discussões com peças cirúrgicas filmadas, enquanto as alterações microscópicas foram desenvolvidas no chamado "Projeto Atlas".

Discussão: Nosso projeto foi bem dinâmico com grande aceitação por parte dos alunos que tiveram atitudes mais proativas, principalmente em relação ao estudo dos casos de microscopia. As estratégias também se prestaram muito bem como forma de avaliação formativa.

Conclusão: Muitas das estratégias que têm sido desenvolvidas por diferentes grupos de professores neste momento da pandemia trouxeram alternativas muito interessantes que devem ser incorporadas ao curso mesmo quando ele voltar a ser presencial. Peças filmadas contemplaram o estudo da macroscopia durante esse período de restrições e podem ser incorporadas à rotina presencial, intercalando com as técnicas utilizadas anteriormente. Já o "Projeto Atlas" foi uma experiência positiva, ressaltando o papel do aluno como protagonista do seu processo de ensino/ aprendizagem e o trabalho em pequenos grupos como pontos principais.

Palavras-chave: Patologia; Microscopia; Educação de Graduação em Medicina; Covid-19.

\begin{abstract}
Introduction: Pathology is a basic discipline that acts as a link between basic and clinical-surgical cycles. Since the beginning of 2020, with the pandemic caused by the severe acute respiratory syndrome coronavirus 2 (Sars CoV-2) and the implementation of emergency remote education (ERE), the pathology course in our institution has undergone changes and adaptations. This paper aims to report the activities developed in our college for the teaching of general pathology, within the context of ERE and discuss how part of these strategies may be incorporated after the end of the pandemic.

Experience report: For teaching macroscopic changes we used discussions with filmed surgical specimens while microscopic changes were developed in the so-called "Atlas Project".

Discussion: Our project was very dynamic and widely accepted by students with a more proactive attitudes, especially regarding the study of microscopic cases. The strategies also lent themselves very well as a formative form of evaluation.

Conclusion: Many of the strategies that have been developed by different groups of teachers during this pandemic have brought very interesting alternatives that should be incorporated into the course even when face-to-face classes are resumed. The filmed pieces include the study of macroscopy during this period of restrictions and can be incorporated into the classroom routine, interspersed with the techniques used previously. The Atlas Project was a positive experience, highlighting the role of the student as the protagonist of his teaching/learning process and work in small groups as main points.
\end{abstract}

Keywords: Pathology; Microscopy; Undergraduate Medical Training; COVID-19.

${ }^{1}$ Faculdade de Ciências Médicas da Santa Casa de São Paulo, São Paulo, São Paulo, Brasil.

Editora-chefe: Rosiane Viana Zuza Diniz.

Editor associado: Antonio da Silva Menezes Junior.

Recebido em 27/08/21; Aceito em 05/10/21.

Avaliado pelo processo de double blind review. 


\section{INTRODUÇÃO}

A patologia é uma disciplina básica em vários cursos de graduação da área da saúde, sobretudo na Medicina. Possui papel primordial no link (interligação) entre as ciências básicas e as disciplinas clínicas, promovendo a base para o entendimento das doenças ${ }^{1,2}$. Para seu ensino, inúmeras técnicas e atividades já foram descritas e utilizadas ${ }^{3}$. Além das aulas expositivas, muitas vezes realizadas com dezenas de alunos simultaneamente, a utilização de aulas práticas contribui muito para a contextualização dos conceitos discutidos e o consequente aprendizado. De forma muito tradicional, a sala de autópsia já demonstrou ser um ambiente extremamente rico para o aprendizado. Sessões de discussão de peças cirúrgicas ou visitas a museus com peças anatomopatológicas contribuem para a análise macroscópica. Laboratórios com microscópios e caixas de lâminas de vidro selecionadas auxiliam no entendimento do processo patológico ${ }^{3,4}$.

Entretanto, o cenário mundial sofreu alterações drásticas a partir do início de 2020, com a pandemia provocada pela síndrome respiratória aguda grave do coronavírus 2 (severe acute respiratory syndrome coronavirus 2 - Sars-CoV-2), causadora da coronavirus disease 2019 (Covid-19). A partir de março de 2020, as instituições de ensino por todo o país tiveram que suspender suas atividades presenciais e passar a atuar de forma remota. Essa transição foi abrupta e sem preparo prévio, numa medida emergencial ${ }^{5-7}$.

A adequação do currículo foi um grande desafio, principalmente com relação às atividades práticas. Em nossa instituição, o conteúdo teórico foi adaptado para aulas virtuais síncronas (via plataformas GMeet e Teams) e assíncronas, muitas vezes acompanhadas de exercícios ou trabalhos para serem desenvolvidos e entregues pelos alunos, por meio do ambiente virtual de aprendizagem (AVA), utilizando a plataforma Moodle. No caso da disciplina de processos patológicos gerais, consideramos que seria necessária uma adaptação que substituísse, com o menor prejuízo possível, as aulas práticas de histologia e de autópsia antes utilizadas no ensino da patologia geral em nossa instituição.

O presente trabalho tem como objetivos relatar as atividades desenvolvidas em nossa faculdade para o ensino da patologia geral, dentro do contexto do ensino remoto emergencial (ERE), e discutir como parte dessas estratégias deverá ser incorporada após o término da pandemia.

\section{RELATO DE EXPERIÊNCIA}

Em nossa instituição, a disciplina de processos patológicos gerais na graduação em Medicina é ministrada no segundo ano do curso e é dividida em quatro blocos: 1. adaptações celulares ao crescimento e à diferenciação celular/lesões celulares reversíveis e irreversíveis, 2. distúrbios circulatórios, 3. processos inflamatórios e 4. neoplasias. Diante da impossibilidade de continuarmos com as aulas na sala de autópsia (necroaula) e nos laboratórios de microscopia, como de costume, desenvolvemos dois projetos distintos.

A identificação das alterações macroscópicas provocadas pelos distintos processos patológicos nos diferentes órgãos e sistemas contribui sobremaneira para o entendimento da fisiopatologia e das consequentes manifestações clínicas das diversas doenças estudadas. Nesse sentido, a utilização de imagens em duas dimensões, disponibilizadas em livros didáticos e sites instrucionais, tem importante papel didático. $\mathrm{Na}$ tentativa de tornar essa percepção ainda mais real, optamos por utilizar alguns vídeos de peças cirúrgicas e de autópsias.

Após a seleção das peças cirúrgicas e de autópsia mais representativas em nosso arquivo, procedemos à documentação de cada uma individualmente. Cada vídeo teve duração de um a três minutos, e todos foram padronizados em fundo azul-escuro. As professoras patologistas que fizeram as gravações manuseavam as peças ou simplesmente apontavam as áreas de interesse com uma pinça cirúrgica. Para cada bloco de conteúdo, produziram-se de oito a 12 vídeos.

Ao final de cada bloco, realizou-se um encontro online síncrono. A turma foi dividida em salas de 40 alunos para cada professora. A sessão teve duração de duas horas e foi constituída pela apresentação dos vídeos e discussão dos processos patológicos envolvidos, e, no encerramento do bloco, revisou-se, de forma aplicada, o conteúdo.

Já para suprir a necessidade do estudo microscópico, a digitalização de lâminas foi a técnica escolhida. Para cada bloco de conteúdo, escanearam-se de dez a 15 lâminas didáticas. A disponibilização desse material se deu por meio da plataforma PathPresenter, acessível remotamente a cada estudante por meio de um link.

A dinâmica para discussão das lâminas foi nomeada "Projeto Atlas". Os alunos foram divididos em pequenos subgrupos (dez alunos) que se reuniam para o estudo por pares, por um período de quatro horas, reservado para a atividade. Os alunos deveriam identificar o órgão representado e o processo patológico envolvido, e elaborar uma breve resenha da fisiopatologia. Foi orientada a documentação de cada caso com imagens ilustrativas e as devidas sinalizações, por meio de setas ou figuras geométricas. Num segundo momento, os alunos se reuniam com o professor responsável para discussão e debate. Cada professor se reunia com quatro subgrupos (de dez alunos cada), e os alunos eram sorteados para apresentar o produto do estudo para o restante da turma, momento em que foram submetidos a uma avaliação formativa. Após esse encontro, o grupo deveria se reunir novamente, com a 
demanda de realizar a revisão do material e a formatação do trabalho final a ser entregue ("Projeto Atlas"). Ao final do curso, o compilado de todas as lâminas estudadas gerou um portfólio, submetido à avaliação. A nota final foi composta pelo conceito emitido diante das apresentações nas sessões síncronas, de caráter formativo, e pela avaliação somativa do trabalho final entregue pelo grupo.

\section{DISCUSSÃO}

Com pouco ou praticamente nenhum tempo para preparo, professores de todo o mundo, de todas as áreas, tiveram que se adequar ao ERE, o que não foi diferente na área da saúde? É importante ressaltar que essa medida difere do ensino a distância (EaD). O ERE é uma situação emergencial e transitória, na qual o currículo é adaptado para atividades remotas. Já o EaD corresponde a um currículo programado previamente para ser executado a distância, de forma permanente ${ }^{8}$.

$\mathrm{Na}$ formação do médico generalista, o ensino da patologia envolve atividades práticas de macro e microscopia, importantes na compreensão e no aprendizado da fisiopatologia das doenças ${ }^{9}$. Com a instalação do ERE, as estratégias de ensino utilizadas deveriam se adequar às necessidades de distanciamento social com restrição de atividades presenciais.

A escolha da produção de vídeos demonstrativos de peças cirúrgicas para exposição e discussão em encontros síncronos foi uma alternativa para as aulas práticas de macroscopia e autópsia. O Departamento de Patologia da Universidade de Chicago relatou experiência semelhante ${ }^{8}$. Realizaram-se diversos tipos de atividades remotas, incluindo discussão de vídeos demonstrativos, trabalhos em pequenos grupos, quizzes, entre outros.

Vídeos macroscópicos podem ser encontrados na internet, desde antes da pandemia. Não se trata de um material numeroso, ainda mais utilizando descritores de busca na língua portuguesa. Nossa instituição, assim como a Universidade de Chicago em seu relato ${ }^{8}$, decidiu produzir seus próprios vídeos, a partir do acervo de peças da disciplina, com o laboratório de patologia assistencial. Assim, o material utilizado para discussão com os alunos foi exclusivo e diretamente associado à demanda regional.

Em nossa experiência, a produção dos vídeos não representou um trabalho de alto custo ou alta complexidade, com o conteúdo básico já gravado. Pretendemos, com a parceria do laboratório assistencial, produzir vídeos de forma contínua, de modo a expandir e atualizar a coleção. Mesmo após a retomada das atividades totalmente presenciais, essas gravações poderão ser usadas para ilustrar aulas expositivas ou em atividades de revisão. Acreditamos, assim, que todo esse trabalho prévio e o esforço em manter a atualização contínua do banco de vídeos trarão grande recompensa na valorização do nosso curso.

Com relação à microscopia, o uso de lâminas escaneadas também já ocorria anteriormente à Covid-19 ${ }^{10-13}$, porém na época nossos alunos tinham contato com as lâminas no momento da aula, no laboratório de informática. Entretanto, a necessidade das atividades remotas alavancou essa prática. $A$ disponibilização prévia das lâminas e o trabalho em pequenos grupos provocaram uma atitude mais ativa dos alunos na busca do conhecimento, trazendo, a cada aula, novos desafios.

O“Projeto Atlas" que desenvolvemos se baseou na análise dos achados microscópicos de casos anatomopatológicos corriqueiros, com o estudo de doenças que fazem parte da rotina do médico generalista. A Faculdade de Medicina da Universidade Johns Hopkins reportou sessões síncronas e assíncronas semelhantes com estudantes da graduação, nas quais eram discutidos casos da rotina do laboratório de patologia, inclusive em tempo real para liberação dos laudos ${ }^{14}$. Essa universidade norte-americana utilizou lâminas escaneadas e transmissão ao vivo de lâminas de vidro, com o auxílio de câmeras acopladas a microscópios de múltiplas cabeças ${ }^{14}$.

Nosso projeto foi bem dinâmico com grande aceitação por parte dos alunos que tiveram atitudes mais proativas, principalmente em relação ao estudo dos casos de microscopia. Anteriormente, a atitude era muito passiva em relação ao aprendizado das alterações microscópicas. Observamos que, além de desenvolver o conhecimento dos processos patológicos em si, foi uma atividade importante para o aprimoramento de habilidades e atitudes. Os estudantes trabalharam de forma cooperativa em pequenos grupos, treinaram o poder de síntese na confecção das apresentações e do portfólio final, praticaram oratória, pois tiveram que explicar seu trabalho para toda a turma, além de estudarem a patologia com foco na fisiopatologia, recordando os conteúdos de anatomia, histologia e fisiologia. As estratégias também se prestaram muito bem como forma de avaliação formativa, mesmo remotamente, o que configura um desafio extra ao professor. Embora muitos alunos tenham mantido câmeras fechadas durante o encontro síncrono, a participação oral foi satisfatória, em grande parte incentivada pelo fato de todos terem sido chamados para expor o trabalho e também por já terem tido, previamente, o momento de estudo em grupo, favorecendo a presença de dúvidas. Ainda não temos claro como será o retorno às atividades presenciais, mas, sem dúvida, pretendemos manter o "Projeto Atlas" na grade, com as adaptações necessárias.

\section{CONSIDERAÇÕES FINAIS}

Muitas das estratégias que têm sido desenvolvidas por diferentes grupos de professores neste momento da pandemia 
trouxeram alternativas muito interessantes que devem ser incorporadas ao curso mesmo quando ele volte a ser presencial. Peças filmadas contemplaram o estudo da macroscopia durante este período de restrições e podem ser incorporadas à rotina presencial, intercalando com as técnicas utilizadas anteriormente. Já o "Projeto Atlas" foi uma experiência positiva, pois ressaltou o papel do aluno como protagonista do seu processo de ensino/aprendizagem e o trabalho em pequenos grupos como pontos principais.

\section{CONTRIBUIÇÃO DAS AUTORAS}

Todas as autoras contribuíram igualmente para a construção do artigo.

\section{CONFLITO DE INTERESSES}

Declaramos não haver conflito de interesses.

\section{FINANCIAMENTO}

Declaramos não haver financiamento.

\section{REFERÊNCIAS}

1. Du Boulay C. Learning pathology: why? how? when? J Clin Pathol. 1997;50(8):623-24

2. Herrmann FE, Lenski M, Steffen J, Kailuweit M, Nikolaus M, Koteeswaran $\mathrm{R}$, et al. A survey study on student preferences regarding pathology teaching in Germany: a call for curricular modernization. BMC Med Educ. 2015;15:94.

3. Bueno FT, Issa YSMM. Técnicas de ensino e atividades em sala de aula para disciplina de patologia na graduação: revisão de literatura. Arq Med Hosp Fac Cienc Med Santa Casa São Paulo. 2018;63(3):215-20.
4. Fenderson BA. Strategies for teaching pathology to graduate students and allied health professionals. Hum Pathol. 2005;36(2):146-53.

5. Appenzeller S, Menezes FH, Santos GG, Padilha RF, Graçal HS, Bragança JF. Novos tempos, novos desafios: estratégias para equidade de acesso ao ensino remoto emergencial. Rev Bras Educ Med. 2020;44(Sup 1):e0155.

6. Rose S. Medical student education in the time of Covid-19. JAMA. 2020;323(21):2131-2.

7. Kaul V, Gallo de Moraes A, Khateeb D, Greenstein Y, Winter G, Chae $\mathrm{J}$, et al. Medical education during the Covid-19 pandemic. Chest. 2021;159(5):1949-60.

8. Fu L, Swete M, Selgrade D, Chan CW, Rodriguez R, Wolniak K, et al. Virtual pathology elective provides uninterrupted medical education and impactful pathology education during the Covid-19 pandemic. Acad Pathol. 2021;28:8.

9. Kirkpatrick CJ. How do we teach pathology? Bridging the gap between theory and practice. J Pathol. 1989;157(2):157-9.

10. Ford JC, Pinder KE, Ovalle WK, Li CH. Pathology education in a multisite urban/rural distributed curriculum. Hum Pathol. 2008;39(6):811-6.

11. Helle L, Nivala M, Kronqvist $P$, Gegenfurtner A, Bjork P, Saljo R. Tradicional microscopy instruction versus process-oriented virtual microscopy instruction: a naturalistic experiment with control group. Diagn Pathol. 2011;6(Suppl 1):S8.

12. Walkowski S, Lundin M, Szymas J, Lundin J. Students' performance during practical examination on whole slide images using view path tracking. Diagn Pathol. 2014;9:208.

13. Leifer $Z$. The use of virtual microscopy and a wiki in pathology education: tracking student use, involvement, and response. J Pathol Inform. 2015;6:30.

14. White MJ, Birkness JE, Salimian KJ, Meiss AE, Butcher M, Davis K, et al. Continuing undergraduate pathology medical education in the coronavirus disease 2019 (Covid-19) global pandemic: the Johns Hopkins virtual surgical pathology clinical elective. Arch Pathol Lab Med. 2021;145(7):814-20 